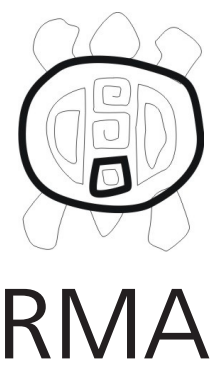

Arqueología

\section{Cuerpos en contraste: reflexiones sobre el tratamiento de los difuntos en dos entierros de 3.000 años A.P. (Valle del Cajón, Noroeste argentino)}

\author{
Leticia Inés Cortés
}

CONICET-Museo Etnográfico "Juan B. Ambrosetti”, Facultad de Filosofía y Letras, Universidad de Buenos Aires. E-mail: leticiacortes@gmail.com

\begin{abstract}
Resumen
Dos contextos funerarios datados en 3.000 años A.P. procedentes del sur del valle del Cajón mostraron interesantes contrastes en cuanto al tratamiento, disposición y asociación de los cuerpos con diversos objetos. El primero es un entierro múltiple donde los cuerpos, entremezclados unos con otros y sin articulación entre sus partes anatómicas se hallaron asociados a una máscara antropomorfa de cobre. El segundo caso se trata de la inhumación de un niño en posición extendida junto a una cuenta de collar lítica y un pendiente de cobre. Los entierros se emplazaban adyacentes uno del otro aunque separados por estructuras de piedra simétricas y opuestas entre sí.

De acuerdo a lineamientos sugeridos por la antropología del cuerpo y los estudios sobre materialidad, se explora una primera interpretación donde los cuerpos constituyen el eje de análisis. El modo en que estos fueron tratados -su individualidad anatómica obliterada o conservada, su asociación con objetos que podrían referir a un colectivo humano, en contraste con otros de uso y demarcación personal, así como la disposición inversa de las estructuras que los contienen- permite proponer que nociones de simetría y oposición habrían estado implicadas en el tratamiento de los difuntos por las poblaciones que habitaron el valle tres mil años atrás.
\end{abstract}

Palabras clave: contextos funerarios; cuerpos; gestos; 3.000 años A.P.; Noroeste argentino

Bodies in contrast: Reflections on the treatment of the dead in two burials of 3,000 years BP (Cajon Valley, Northwest Argentina)

\begin{abstract}
Two funerary contexts dated to 3.000 years B.P. from the southern Cajón Valley showed interesting contrasts as far as the treatment, disposition and association of the bodies with diverse objects is concerned. The first one is a multiple internment where several bodies were laid out mixed with one another without any anatomical articulation. These were associated to an anthropomorphic copper mask. The second one is the inhumation of a child in an extended position associated to a lithic bead and a copper pendant. Both contexts were set adjacent to each other though separated by symmetrical and opposed stone structures.

Along with some perspectives suggested by the anthropology of the body and studies of materiality, I explore a first interpretation in which bodies are considered the axis of the analysis. The way bodies were treated-the obliteration or conservation of their anatomy, their association with objects that could refer to a human collective in contrast to those of personal use and demarcation, as well as the inverse disposition of the structures that contained them, allows to think that notions of symmetry and opposition would have been implied in the treatment of the deceased by those who inhabited the valley three thousand years ago.
\end{abstract}

Keywords: funerary contexts; bodies; gestures; 3000 years BP; Northwestern Argentina

\section{El gesto dado: acerca de la disposición de los cuerpos de los difuntos}

Así como durante la vida las personas usan y modifican sus cuerpos de formas culturalmente prescriptas -formas que transmiten sentido de pertenencia, creencias, sentimientos, historias de vida- tras la muerte, los cuerpos continúan siendo 'entidades plásticas' que requieren y exigen manipulación por parte de los vivos (Meskell 2004: 124). Tal como el modo de caminar, pararse, descansar o dar a luz son formas culturalmente determinadas de movimiento y postura (e.g. Bremmer y Roodenburg 1991; Kendon y Blakely 1986; Mauss 1979), también la disposición de los cuerpos de los difuntos -el gesto que les es dado- y las acciones que toman lugar antes, durante y con posterioridad al entierro responden a conceptualizaciones históricamente específicas. Varios ejemplos etnográficos de distintas regiones de los Andes dan cuenta de estas prácticas y sus resultados (ver Aláez García 2001; Allen 1982, 1988: 54-63; Bastien 1978: 171- 
187, 1995; de Hoyos 2001; Martínez 2008; Pærregaard 1987). Entre los Laymi de Bolivia, por ejemplo, se ata el cuello, los brazos y pies del muerto a fin de lograr restringir el espíritu a su propio cuerpo, evitando así que cause daños a los vivos (Harris 1982: 53). Estas acciones podrían asimilarse a lo que Mauss ha denominado 'técnicas del cuerpo' implicando por ello que los gestos poseen -al igual que cualquier acción mágica, religiosa o simbólicauna efectividad (Mauss 1979: 104). En la misma línea de pensamiento, estudios sobre la semiótica del gesto han resaltado que, aunque tiendan a ser usualmente identificadas con lo inmaterial, las expresiones gesturales tienen un resultado, producen consecuencias, y es allí donde su eficacia radica en última instancia (Schmitt 1991: 62). Pero mientras que los gestos actuados por los vivos son efectivos y transformadores, también son efímeros y pasajeros. En contraste, los gestos dados a los difuntos pueden estar destinados a perdurar en el tiempo al igual que los efectos que ellos propician (Cortés 2007).

Desde una mirada que considere al cuerpo como eje del análisis del registro arqueológico de contextos funerarios, la perspectiva del gesto se vuelve una herramienta válida para comenzar a pensar sobre el modo en que los cuerpos fueron tratados y la forma en que tales acciones refieren a concepciones históricamente específicas de la vida y la muerte. Si la eficacia de los gestos radica en su habilidad para regular el comportamiento de los otros en una relación interpersonal (Kendon 1981: 141), entonces este acercamiento podría ser fructífero en su aplicación al entendimiento de la interacción entre vivos y muertos en el pasado andino prehispánico (ver por ejemplo, Allen 1988: 57-59; Sillar 1992, 1996). En este sentido, esta perspectiva atiende a la necesidad de historización y problematización de los cuerpos (Hamilakis et al. 2002: 4; Pluciennik 2002: 177), buscando superar la arbitraria separación entre lo natural y lo cultural, a partir de la cual el cuerpo humano es visto primero como una entidad biológica universal y pre-social sobre la que luego se imprimen significados culturales (Fowler 2002: 47; Hamilakis et al. 2002: 4; Ingold 2000: 173; Thomas 2007: 214). En todo caso los cuerpos son biología y cultura, una y otra al mismo tiempo. Los cuerpos - presentes y pasados- son materia de significaciones históricas y en tal sentido deben ser considerados. Esta postura es inseparable de una crítica reflexiva sobre las categorías a través de las cuales son pensados (categorías que, por otro lado, tienen consecuencias directas sobre las cuestiones éticas que se derivan de su estudio y análisis) y del reconocimiento en este mismo movimiento, de nuestro lugar como investigadores situados (ver Hamilakis et al. 2002). Asimismo, personas y objetos se implican mutuamente, por lo que el entendimiento conjunto de unos y otros se vuelve ineludible (Hoskins 1998: 2). La naturaleza imbricada de la gente y las cosas, inseparables ellas mismas del tiempo y el espacio que evocan (ver Lazzari 2005) es la materia que constituye y da forma a los sistemas de referencia que son históricamente específicos. En otras palabras, las materias de las cuales están hechos los mundos, están hechas a la vez que esos mismos mundos (Goodman 1990: 24).

Considerando algunos aspectos de la antropología del cuerpo y los estudios de materialidad en arqueología, a continuación se explora una posible interpretación sobre dos contextos de enterratorio procedentes del valle del Cajón datados en 3000 años AP, atendiendo principalmente a las semejanzas y contrastes que ambos denotan.

\section{Dos entierros en lo alto del bordo}

Los contextos de entierro a los que me referiré en este trabajo proceden de la localidad de La Quebrada ubicada al sur del valle del Cajón (departamento de Santa María, Catamarca) (Figura 1). Como sucede a menudo en el Noroeste argentino, esta localidad presenta un vasto y variado registro de las antiguas poblaciones que habitaron el Valle. A fin de conocer las particularidades en su historia de ocupación, a lo largo de los últimos años se han realizado extensas investigaciones en el área (Gero ms; Scattolin et al. 2007, 2009a, 2009b). Como producto de estas investigaciones se han detectado una

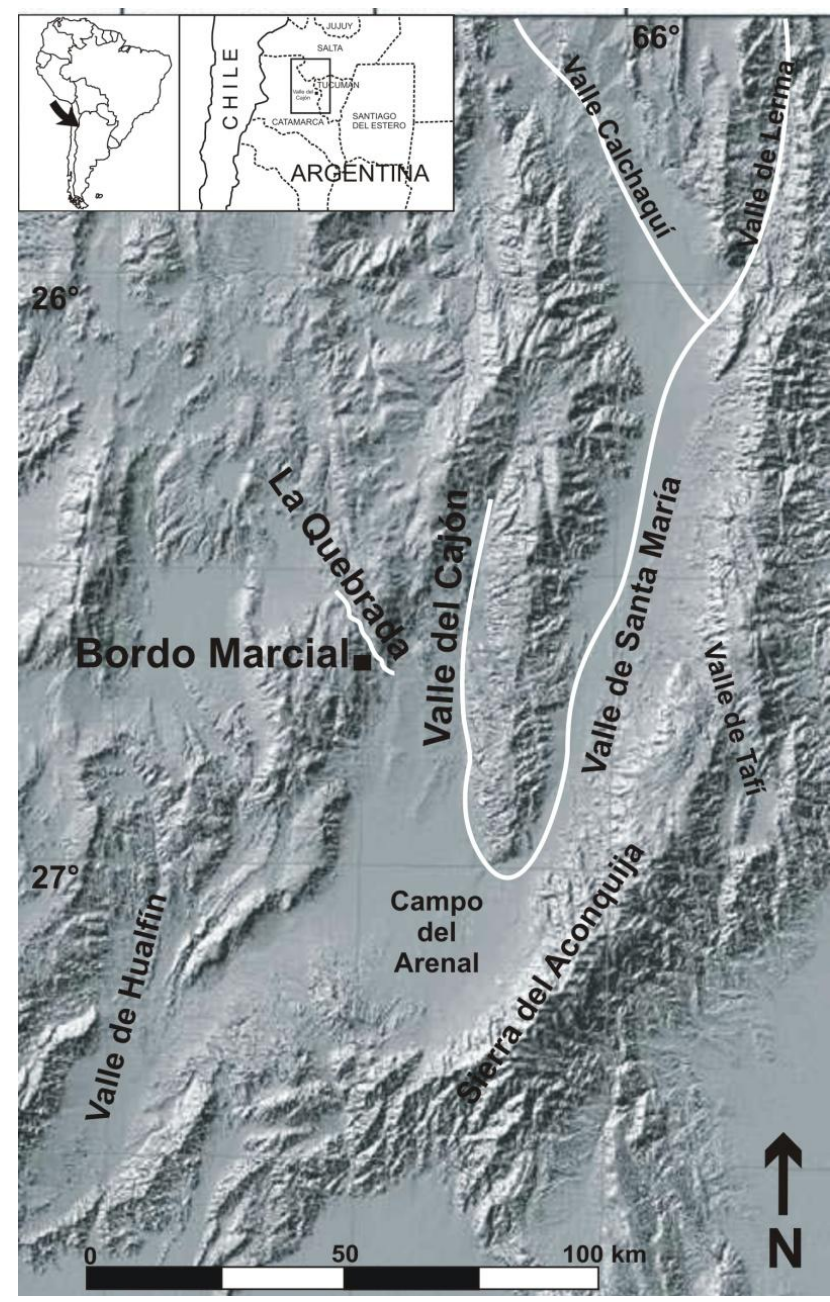

Figura 1: Ubicación del área de estudio dentro del Noroeste argentino. 
serie de sitios de habitación, estructuras agrícolas y varios contextos de entierro de características y temporalidad diversa. En este lugar, me ocuparé principalmente de dos de ellos: el Contexto 1 y el Contexto 2 de Bordo Marcial, denominación que ha sido dada por hallarse ambos en la parte alta del bordo sobre el cual se extiende la aldea formativa homónima.

Durante la temporada de campaña de octubre de 2005, mientras realizábamos una prospección del área, fuimos advertidos por los pobladores locales acerca de la presencia de restos humanos que habían aflorado en superficie tras el paso de las lluvias en abril de ese mismo año. El sector donde se hallaban los huesos correspondía a la parte más alta de un bordo que se extiende en altitud y longitud en sentido Este-Oste surcando un cerro de mayor altura (Figura 2). Sobre la parte baja de este bordo se distribuyen una serie de estructuras de piedra subcirculares que conforman el sitio formativo de Bordo Marcial, una de las cuales ha sido datada en 1800 años antes del presente. En la foto aérea puede distinguirse asimismo que al norte de las estructuras de habitación existe un área de suelo medanoso que, por las evidencias halladas en superficie (cuentas de collar, huesos fragmentarios, tiestos cerámicos), se trate muy probablemente un cementerio (ver Figura 2). El sector dónde se emplazaban los Contextos 1 y 2 de Bordo Marcial corresponde a un lugar particularmente destacado del paisaje, desde donde hoy se obtiene una visión panorámica de las estructuras de habitación así como del área circundante: la sierra del Cajón extendiéndose al este, y el campo del Arenal, al sur.

En lo alto del bordo, una gran cantidad de fragmentos de huesos y dientes se hallaban dispersos en superficie, muy deteriorados y blanqueados por la exposición a la intemperie. Asimismo, algunas piedras planas de formas más o menos regulares yacían en el suelo mientras que otras se encontraban aún clavadas en la tierra. Estas últimas, indicio de una estructura semi-enterrada,

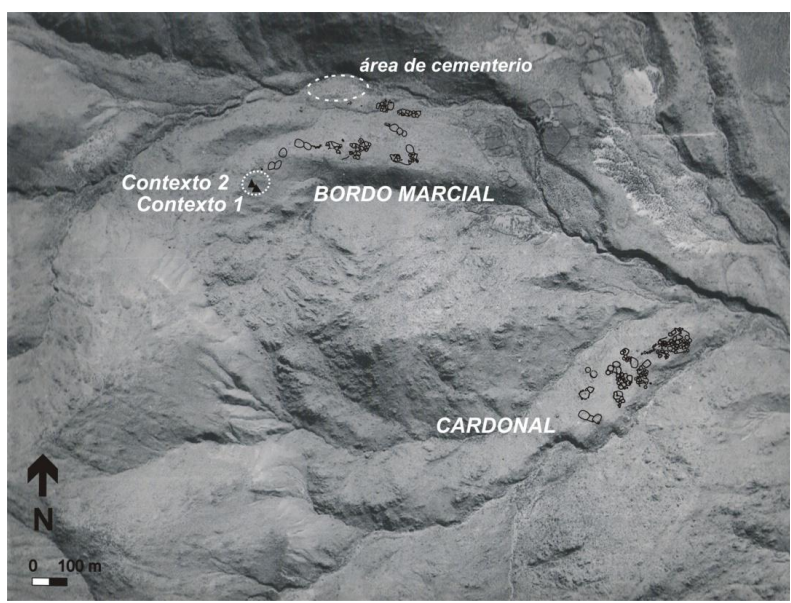

Figura 2: Foto aérea señalando la ubicación de los Contextos 1 y 2 de Bordo Marcial. Contexto 1: Latitud S 26 40' 29.2", Longitud W $66^{\circ}$ $28^{\prime}$ 39.1", Altitud 3194 msnm. Contexto 2: Latitud S 26 40' 29.3", Longitud W 66²8' 39.3", Altitud 3184 msnm. formaban una única pared curva con una leve concavidad hacia el Este de aproximadamente 1,5 m de extensión en sentido Norte-Sur. Este era el Contexo 1 de Bordo Marcial.

La excavación de rescate descubrió una gran cantidad de restos óseos humanos circunscriptos a un área aproximada de $2 \times 1,20 \mathrm{~m}$, entremezclados unos con otros sin articulación entre ellos (Figura 3 ). Posteriormente se determinó que correspondían a un número mínimo de 14 individuos, 7 adultos -con representación de ambos sexos- y 7 subadultos. Entre estos últimos se identificaron un niño de edad aproximada entre 2-3 años, otro de entre 3-4 años, dos de entre 4-5 años; uno de entre 8-10 años, otro de aproximadamente 10-12 años y por último un individuo de entre 14-16 años de edad'.

La remoción de los restos óseos fue muy dificultosa debido a que el sedimento que los contenía era extremadamente duro y compacto. Una vez recuperados, se observó que las piedras que formaban la estructura estaban calzadas en el sedimento por debajo del nivel de los huesos. Las de mayor tamaño estaban a su vez sostenidas por otras piedras más pequeñas colocadas del lado interno. En la elección de las piedras principales parece haberse priorizado las formas chatas, tipo laja, de las variantes naturales disponibles localmente. Dos de las piedras que se hallaban sueltas en superficie parecen además haber sido levemente retocadas para lograr una forma rectangular alargada, manteniendo una de sus caras plana y la otra algo redondeada.

Al momento de detectar este enterramiento, los pobladores hallaron un objeto de metal semienterrado que fue retirado antes de nuestra llegada: una máscara antropomorfa realizada sobre una fina lámina de cobre (Figura 4). Durante la excavación se recuperaron algunos fragmentos óseos que presentaban la coloración verde característica del contacto con el carbonato de cobre, evidencia independiente de la asociación entre los restos humanos y la máscara. Por sus características, se trata de una pieza única para el Noroeste argentino y el área andina (ver Scattolin et al. 2010).

Sus dimensiones son $180 \mathrm{~mm}$ de alto, $150 \mathrm{~mm}$ de ancho y $1 \mathrm{~mm}$ de espesor, y muestra una leve convexidad. Los rasgos antropomorfos fueron logrados mediante la

\footnotetext{
${ }^{1}$ La estimación de edad en los subadultos se realizó a partir del grado de desarrollo dental según el método de Moorees et al. (1963) y Ubelaker (1989). En el caso de los individuos adultos, no fue posible establecer rangos de edad debido a la ausencia o deterioro de partes diagnósticas. La estimación de sexo en adultos también se vio comprometida por la fragmentación y deterioro de las partes esqueletales diagnósticas (cráneos y pelvis). No obstante, considerando la variación morfológica existente en las apófisis mastoideas del hueso temporal, puede inferirse la presencia de individuos de ambos sexos en la muestra (Buikstra y Ubelaker 1994:15-21). No se estimó el sexo en subadultos por tratarse de individuos en etapa de desarrollo que aún no manifiestan claramente el dimorfismo sexual a nivel esqueletal (Buikstra y Ubelaker 1994:16, Bass 1995 [1971]:25-6, Scheuer y Black 2000:15).
} 

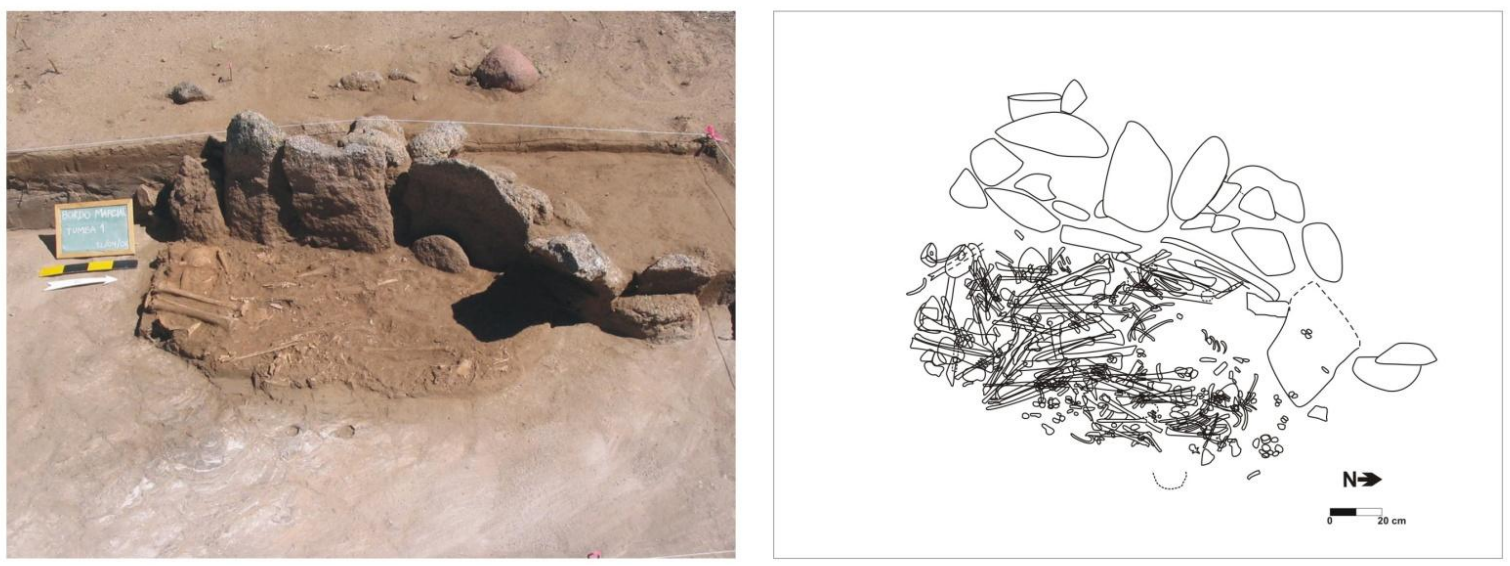

Figura 3: Foto y esquema del Contexto 1 de Bordo Marcial.

técnica altorelieve aplicado desde el revés de la pieza. La boca está conformada por una abertura central rodeada de un relieve horizontal de forma rectangular con vértices redondeados. La nariz cuyo realce es más notorio, se encuentra fracturada probablemente como consecuencia de procesos postdepositacionales. Los ojos son dos orificios oblongos horizontales apenas contorneados en relieve. Posee nueve pequeñas perforaciones circulares en los bordes, dos en cada costado, dos en las esquinas superiores, dos en la parte central del borde superior y uno en la parte central del borde inferior. Los análisis metalúrgicos realizados sobre esta pieza determinaron que la misma fue manufacturada sobre una lámina de cobre mediante la técnica de recocido y martillado (Scattolin et al. 2007-2008, 2010). El Contexto 1 de Bordo Marcial, entonces, era una tumba colectiva rodeada por una pared de piedras chatas dispuestas con una leve concavidad, como conteniendo los restos en su interior. Junto con ellos, una máscara antropomorfa de metal fue depositada. Este contexto fue fechado por AMS en 3001 \pm 49 A.P. (AA82256).

Durante su excavación, tras realizar una segunda ampliación hacia el Oeste, se observó otro alineamiento de piedras que se extendía de manera paralela al anterior. Una nueva ampliación confirmó la presencia de una pared de piedras chatas algo más pequeñas, dispuestas simétricamente aunque en sentido opuesto a las del Contexto 1. A este contexto hemos denominado Contexto 2. A poco más de un metro de la pared de piedras yacía el esqueleto de un individuo subadulto en posición extendida, decúbito dorsal, con la cabeza orientada hacia el Norte y los pies hacia el Sur. Bordeando el costado derecho del cuerpo se detectaron los límites de una pequeña fosa (Figura 5). Se aproximó la edad de este individuo entre los 8 y 12 años $^{2}$.

En asociación a los restos humanos se recuperó una cuenta de collar de piedra cilíndrica de tono verde claro (Figura 6a). Algo más alejado del cuerpo, se halló un pendiente de cobre (Figura 6b). Del sector comprendido entre los restos humanos y la pared de piedras, se recuperaron

\footnotetext{
${ }^{2}$ La estimación de edad se realizó a partir del grado de desarrollo dental según el método de Moorees et al. (1963) y Ubelaker (1989). No se estimó sexo por tratarse de un individuo en etapa de desarrollo que aún no manifiesta claramente el dimorfismo sexual a nivel esqueletal (Buikstra y Ubelaker 1994:16, Bass 1995 [1971]:25-6, Scheuer y Black 2000:15).
}

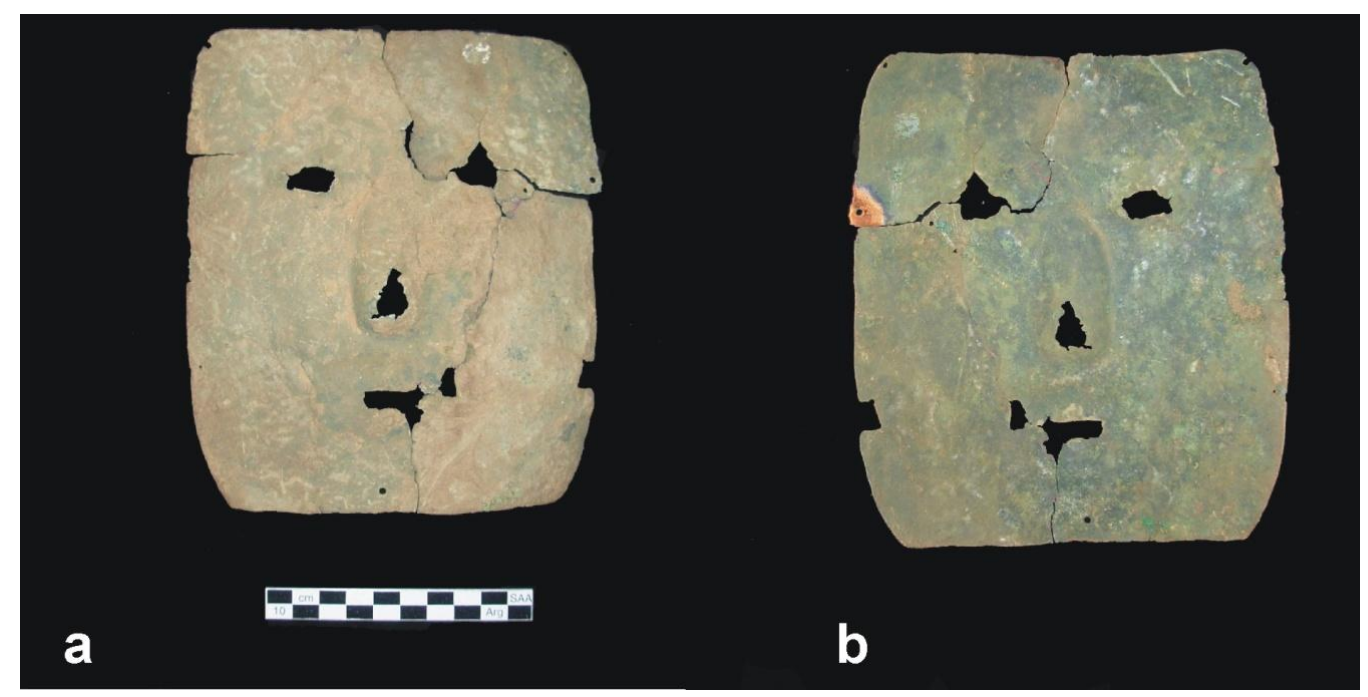

Figura 4: Máscara antropomorfa de cobre asociada al Contexto 1 de Bordo Marcial. a) frente, b) revés. 

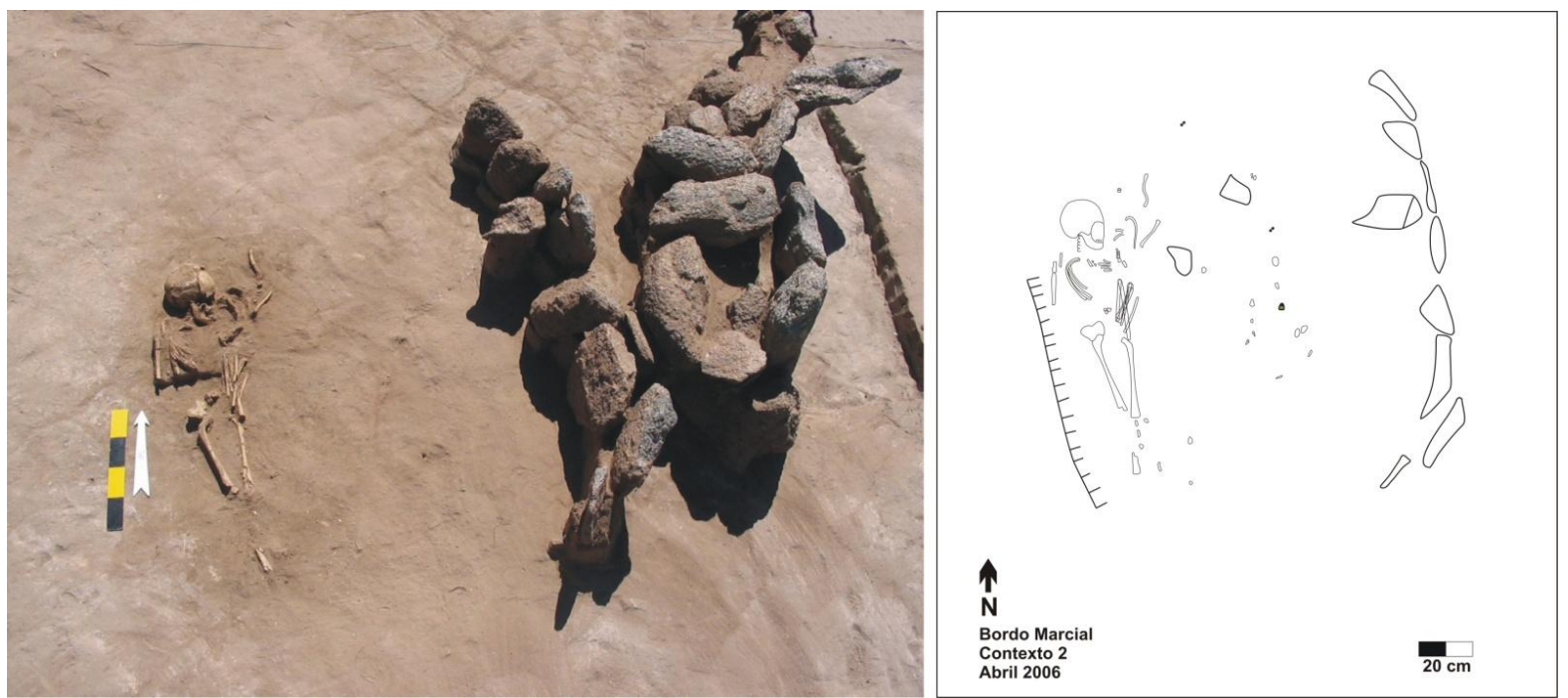

Figura 5: Foto y esquema del Contexto 2 de Bordo Marcial (en la foto se puede observar la disposición simétrica e inversa de ambas estructuras: a la derecha se observa la pared del Contexto 1, a la izquierda la pared del Contexto 2).

además algunos fragmentos óseos y dientes aislados que luego se determinó pertenecieron a al menos otros tres individuos. Se trata de un adulto representado por unas pocas piezas dentales con un grado muy avanzado de desgaste, otro individuo de edad no determinada aunque probablemente adulto y un tercer individuo subadulto de pocos meses de edad del cual sólo se recuperaron un pequeño fragmento de mandíbula y dos gérmenes de molar deciduo. La presencia de restos aislados de estos tres individuos junto al entierro del niño podría haber estado relacionada con el uso cíclico de este lugar para entierros, y que sus restos hubieran sido trasladados en algún momento anterior o posterior al del niño.

El Contexto 2 de Bordo Marcial correspondió así a la inhumación de un individuo que yacía extendido asociado a una cuenta lítica y un pendiente metálico. Un primer molar inferior izquierdo permanente del niño fue fechado en $3057 \pm 50$ A.P. (AA82257).

La similitud de ambos fechados $(3001 \pm 49$ y $3057 \pm 50$
A.P.) permite plantear su contemporaneidad en términos estadísticos, esto es, que ambas tumbas se solapan en el tiempo, permitiendo suponer que aunque no fuesen estrictamente contemporáneas, una de ellas ya estaba presente al momento de la construcción de la segunda. Por el momento, no se ha detectado ningún otro entierro o registro de habitación de esta antigüedad en el paisaje circundante.

\section{Gestos de simetría y contraste}

Tal vez, la primera idea que transmite el Contexto 1 de Bordo Marcial, sea aquella de contraste. Aquél generado por un lado, por el conjunto de los cuerpos entremezclados, confundidos unos con otros, no reconocibles individualmente y, por otro, por la presencia de una única forma antropomorfa representada en los rasgos humanos de la máscara.

Esta particular disposición de los cuerpos podría pensarse como tendiente hacia la obliteración del

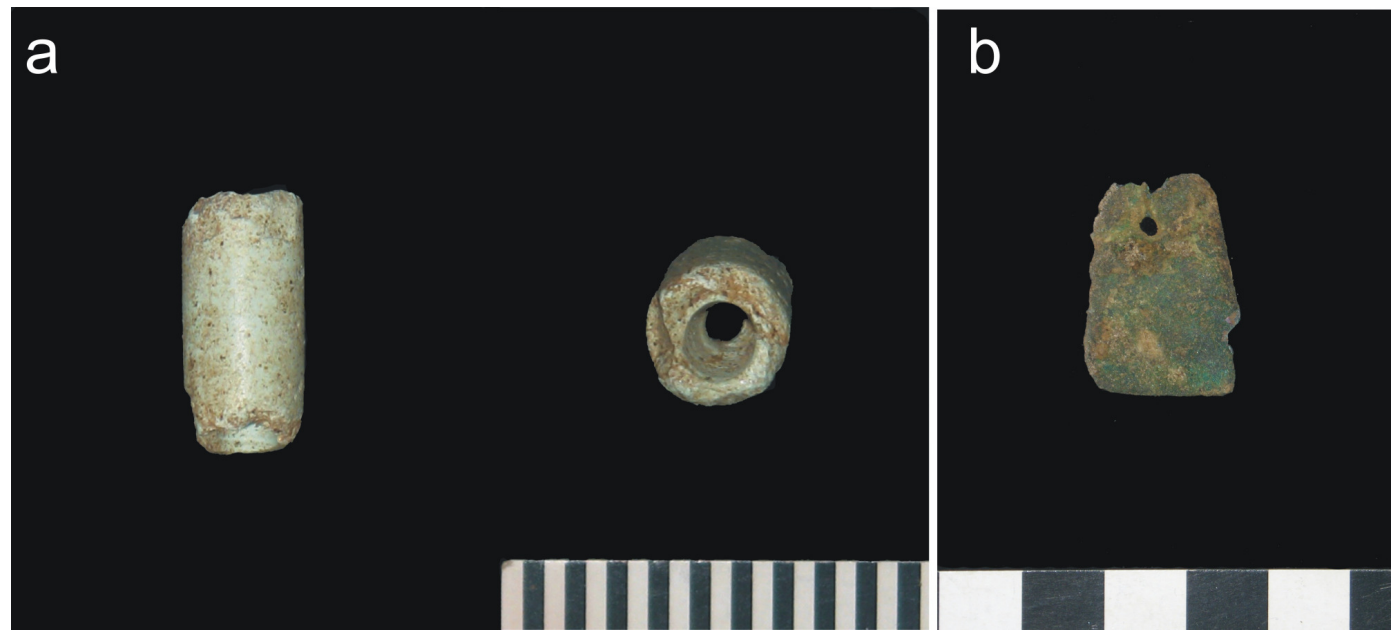

Figura 6: Cuenta lítica (a) y pendiente de cobre (b) asociados al Contexto 2 de Bordo Marcial. 
gesto humano; como la disolución anatómica de las al menos catorce personas que sólo fueron posteriormente reconocibles individualmente a través de la identificación bioarqueológica. En tal sentido, la supresión de los cuerpos individuales de cada una de las personas allí enterradas contrasta con la unicidad del gesto humano de la máscara que acompañaba el conjunto.

Los rasgos plasmados en el metal de la máscara denotan claramente un rostro antropomorfo, cualidad que fue lograda mediante la representación sintética pero efectiva de los ojos, nariz y boca. No obstante, aún cuando la cara suele simbolizar la individualidad tal vez más efectivamente que otra parte del cuerpo (Synnott 1993:73), no han sido incluidos en este rostro de metal otras particularidades que pudieran singularizarlo como hombre o mujer, como una persona adulta o joven, etc. En tal sentido, al menos en su soporte metálico, carece de rasgos como peinados o vello facial, atavíos, adornos o marcas particulares como por ejemplo, aros, tocados o tatuajes. La máscara posee, en este sentido, cierta 'neutralidad' en su humanidad representada. Sin embargo, no es neutral en su expresión, en su gesto: como otras máscaras provenientes de distintos lugares del Noroeste argentino (ver Scattolin et al. 2010), los ojos y la boca están literalmente 'abiertos' por las perforaciones efectuadas sobre el metal.

En su estudio sobre los Kayapo, Turner (1995) ha planteado que las aperturas practicadas por la perforación de las orejas y labios, lejos de tener una finalidad exclusivamente ornamental, están directamente relacionadas con la necesidad de inaugurar en los individuos la habilidad de comunicación en el ámbito social. Esta habilidad es otorgada en determinado momento del desarrollo de cada persona que se incorpora paulatinamente a las actividades sociales. Así, la perforación de las orejas de los infantes implica un acto simbólico de socialización mediante el cual se da al individuo la capacidad de recibir información fundamental y por tanto, de participar activamente en las relaciones de grupo. Lo mismo sucede con la apertura de agujeros en el labio inferior de los hombres adultos a partir de la colocación de discos de diámetros sucesivamente mayores, acción que simbólicamente activa su habilidad para transferir conocimientos a través de la oratoria pública (Turner 1995: 154). Ambos tipos de aperturas son entendidas como formas de 'amplificar' el poder de la comunicación social, ya sea transmitiéndola (en el caso de los discos labiales) o recibiéndola (en el caso de las perforaciones de las orejas) (Turner 1995). Este ejemplo etnográfico es válido en tanto permite comenzar a reflexionar sobre el gesto dado a la máscara como un gesto 'vivo', marcando un nuevo punto de contraste con el contexto de muerte en el cual fue depositada. Tal vez estas aperturas confirieran simbólicamente al personaje representado por la máscara la posibilidad de 'ver' y 'hablar' por el conjunto de las personas que allí fueron enterradas, unificando a través de esta cualidad la desunión de los al menos catorce cuerpos que se funden y se sintetizan en un conjunto homogéneo, indiferenciado.

El Contexto 1 de Bordo Marcial presenta entonces ciertos aspectos de oposición entre la forma en que esta multiplicidad de cuerpos fueron dispuestos y las cualidades del único objeto asociado. Interesantemente, estos contrastes se reafirman y se vuelven aún más significativos al observarlos en relación con el contemporáneo Contexto 2.

Partiendo nuevamente de una mirada desde los cuerpos, se destaca del segundo contexto funerario la anatomía de un único individuo, un niño que yace extendido sobre su espalda, mirando al Este. Asimismo, a través del tipo particular de objetos hallados en este contexto -la cuenta de collar y el pendiente- se refuerza la idea de individualidad transmitida por el gesto dado al cuerpo. Cuando utilizo el término 'individualidad' sin embargo, no estoy haciendo referencia a la 'ideología del individualismo' en términos modernos (ver Brück 2004: 309), ni contraponiéndolo con la 'idea de comunalidad'. Entre los ya mencionados Kayapo, el uso de determinados atavios u objetos de adorno va modificándose a lo largo de la vida de cada individuo, marcando en cada momento la etapa social que atraviesa aquél que lo porta (desde el crecimiento fisiológico, a la iniciación en el mundo adulto, aptitud para casarse o tener hijos, duelo, viudez, etc.). Brazaletes, cuentas, collares, suelen tener un carácter de demarcador social confiriendo y habilitando a la persona que los lleva un determinado lugar en la sociedad (Turner 1995: 158). En este sentido, el valor de los objetos de uso personal radica justamente en la inclusión del individuo como miembro de un grupo. La 'individualidad' resaltada en el Contexto 2 es entendida entonces en términos relacionales (Brück 2004: 311). Los objetos de uso personal, por tanto, son una metáfora del grupo, pero ciertamente diferente a aquella aludida a través de la máscara.

Así entonces, los Contextos 1 y 2 de Bordo Marcial sugieren ideas contrastantes. Este carácter de oposición parece ser realzado y reafirmado por la disposición simétrica aunque exactamente inversa de las estructuras que delimitan cada contexto. En efecto, cada pared de piedras se dispone en forma levemente cóncava, conteniendo y a la vez separando los cuerpos contenidos. La imagen especular que las estructuras reflejan (ver Figura 5) continúa proyectando en su interior otras simetrías invertidas: la de los cuerpos indiferenciables y la de un cuerpo particular; la de la máscara antropomorfa y la de los objetos de uso individual. De esta forma, cuerpos, objetos y estructuras revisten un doble movimiento de simetría y contraste, incluyéndose y excluyéndose mutuamente.

\section{Reflexiones finales}

"...la historia del orden de las cosas sería la historia de lo Mismo -de aquello que, para 
una cultura, es a la vez disperso y aparente $y$ debe, por ello, distinguirse mediante señales y recogerse en las identidades." (Foucault 1968 [1966]: 9)

Las historias de las personas y las historias de los objetos no pueden ser contadas separadamente, porque unas se imbrican con otras inevitablemente (Hoskins 1998: 2). Las distintas materialidades asociadas a los Contextos 1 y 2 de Bordo Marcial transmiten contraste y oposición, pero en tanto esa oposición se construye a partir de una simetría inversa, ambos contextos se aluden uno al otro, y en tal sentido no son ajenos a una misma concepción histórica de la muerte. Desde la mirada occidental podría pensarse que ambos contextos operan una 'distorsión de la clasificación' (Foucault 1968 [1966]: 4). Sin embargo, ambos deberían considerarse formas antagónicas pero no contradictorias, cuyo anclaje radica en una misma cosmovisión, en un mismo 'sistema de referencia' (en el sentido de Goodman 1990: 19). Se trata de una misma lógica clasificatoria que admite ciertas formas y las expresa en términos opuestos. Ambas formas gesturales, la conservación de la integridad corporal y la disolución de ésta (Brück 2004: 310) nos hablan en última instancia, de un sistema de clasificación que reúne a partir de una variedad infinita de posibilidades, formas que se oponen y se implican mutuamente. Si "los procesos de ordenación son parte de la construcción de mundos al igual que lo son los de composición, descomposición y ponderación" (Goodman 1990: 33), los Contextos 1 y 2 de Bordo Marcial representan un orden y ese orden sólo cobra sentido en el mundo que construyeron aquellos que habitaron el sur del valle del Cajón hace 3.000 años.

Buenos Aires, 15 de junio de 2010

\section{Agradecimientos}

Quiero agradecer a los pobladores de La Quebrada por su generosidad y hospitalidad durante mis estadías de trabajo de campo en esta localidad. En particular, a la familia Chaile-Marcial por haber donado la máscara al Museo Eric Boman de Santa María y por permitirnos realizar las labores dentro de su propiedad.

Estos trabajos fueron posibles gracias al esfuerzo conjunto del equipo que dirige María Cristina Scattolin a quien agradezco muy especialmente su guía y apoyo en mi investigación. Agradezco asimismo a los colegas y estudiantes que colaboraron en las excavaciones arqueológicas.

Las observaciones y sugerencias realizadas por los evaluadores fueron de gran utilidad en el mejoramiento de este artículo. De más está decir que los errores son enteramente mi responsabilidad.

\section{Bibliografía}

Aláez García, A. 2001. Duelo andino: sabiduría y elaboración de la muerte en los rituales mortuorios. Chungará, 33(2): 173-178.

Allen, C. 1982. Body and soul in Quechua thought. Journal of Latin American Lore, 8(2): 179-195.

Allen, C. 1988. The hold life has. Coca and cultural identity in an Andean community, Smithsonian Institution Press, Washington.

Bass, W.M. 1995 [1971]. Human Osteology. A Laboratory and Field Manual. Special Publication $N^{\circ} 2$. Missouri Archaeological Society, Columbia.

Bastien, J.W. 1978. Mountain of the condor. Metaphor and ritual in an Andean Ayllu, West Publishing Co, New York.

Bastien, J.W. 1995. The mountain/body metaphor expressed in a Kaatan funeral. Dillehay, T. (ed) Tombs for the living: Andean mortuary practices, 355-378, Dumbarton Oaks, Washington D.C.

Bremmer, J. y H. Roodenburg (eds). 1991. A cultural history of gesture, Polity Press, Cambridge.

Brück, J. 2004. Material metaphors. The relational construction of identity in Early Bronze Age burials in Ireland and Britain. Journal of Social Archaeology, 4(3): 307-333.

Buikstra, J.E., y D.H. Ubelaker. 1994. Standards for data collection from human skeletal remains. Arkansas Archaeological Survey Research Series No. 44.

Cortés, L.I., 2007. "Material Confines. Conceptualizations of death through the materiality of burial structures". Tesis de Maestría. Departamento de Arqueología, Universidad de Sheffield, Inglaterra. 83 páginas. Departamento de Arqueología, Universidad de Sheffield, Inglaterra. Ms.

de Hoyos, M. 2001. Saliendo del Cajón por el Río Jordán: costumbres funerarias del Valle del Cajón, Catamarca, Argentina. Chungará, 33(2): 249-252.

Foucault, M. 1968 [1966]. Las palabras y las cosas. Una arqueología de las ciencias humanas, Siglo XXI Editores, Buenos Aires.

Fowler, C. 2002. Body parts: personhood and materiality in the earlier Manx Neolithic. Hamilakis Y., M. Pluciennik y S. Tarlow (eds), Thinking through the body. Archaeologies of corporeality, pp. 47-69, Kluwer Academic/Plenum Publishers, New York.

Gero, J.M. Registros de campo de las excavaciones en el sitio Cardonal (Valle del Cajón, Catamarca). Ms. 
Goodman, N. 1990. Maneras de hacer mundos. Visor, Madrid.

Hamilakis, Y., M. Pluciennik y S. Tarlow. 2002. Introduction. Hamilakis Y., M. Pluciennik y S. Tarlow (eds), Thinking through the body. Archaeologies of corporeality, pp.1-21, Kluwer Academic/Plenum Publishers, New York.

Harris, O. 1982. The dead and the devils among the Bolivian Laymi. Bloch, M. y J. Parry (eds) Death and the regeneration of life, 2: 45-73, Cambridge University Press, Cambridge.

Hoskins, J. 1998. Biographical objects. How things tell the story of people's lives, Routledge, New York.

Ingold, T. 2000. Building, dwelling, living: how animals and people make themselves at home in the world. Ingold, T. The perception of the environment. Essays in livelihood, dwelling and skill, 10, 172-188, Routledge, London.

Kendon, A. 1981. Geography of gesture. Semiotica, 37 (1/2): 129-163.

Kendon, A. y T.D. Blakely (eds). 1986. Approaches to gesture. Semiotica, Special Issue 62 (1/2).

Lazzari, M. 2005. The texture of things: objects, people, and landscape in Northwest Argentina (First Millennium A.D.). Meskell, L. (ed) Archaeologies of materiality, 6, 126-161, Blackwell, Oxford.

Martínez, B. 2008. Relaciones recíprocas entre vivos y muertos en San Antonio del Cajón. Hidalgo C. (comp.) Etnografías de la muerte, EUDEBA, Buenos Aires (en prensa).

Mauss, M. 1979. Sociology and psychology, Routledge \& Kegan Paul, London.

Meskell, L. 2004. Object worlds in ancient Egypt. Material biographies past and present. Berg, Oxford.

Moorees, C.F.A., E.A. Fanning y E.E. Hunt. (1963). Formation and resorption of three deciduous teeth in children. American Journal of Physical Anthropology 21: 205-213.

Pærregaard, K. 1987. Death rituals and symbols in the Andes. Folk, 29: 23-42.

Pluciennik, M. 2002. Introduction. Hamilakis Y., M. Pluciennik y S. Tarlow (eds), Thinking through the body. Archaeologies of corporeality, pp.173-178, Kluwer Academic/Plenum Publishers, New York.

Scattolin, M.C., L.I. Cortés, C.M. Calo, L. Pereyra
Domigorena y J. Izaguirre. 2007-2008. Una máscara metálica del valle del Cajón, Catamarca, Argentina. Arqueología, 14: 229-237.

Scattolin, M.C., L. Pereyra Domingorena, L.I. Cortés, M.F. Bugliani, C.M. Calo, A.D. Izeta y M. Lazzari. 2007. Cardonal: una aldea formativa entre los territorios de valles y puna. Cuadernos, 32: 211-225.

Scattolin, M.C., L.I. Cortés, M.F. Bugliani, C.M. Calo, L. Pereyra Domingorena, A.D. Izeta y M. Lazzari. 2009a. Built landscapes of everyday life: a house in an early agricultural village of northwestern Argentina. World Archaeology, 41(3): 296-414.

Scattolin M.C., M.F. Bugliani, L.I. Cortés, C.M. Calo, L. Pereyra Domingorena y A.D. Izeta. 2009b. Pequeños mundos: hábitat, maneras de hacer y afinidades en aldeas del Valle del Cajón, Catamarca. Relaciones de la Sociedad Argentina de Antropología, XXXIV: 271-274.

Scattolin M.C., M.F. Bugliani, L.I. Cortés, L. Pereyra Domingorena y C.M. Calo. 2010. Una máscara de cobre de 3000 años. Estudios arqueometalúrgicos y comparaciones regionales. Boletín del Museo Chileno de Arte Precolombino. Publicación del Simposio Estudios sobre minería y metalurgia en los Andes Meridionales, $53^{\circ}$ Congreso Internacional de Americanistas. Enviado.

Schmitt, J. 1991. The rationale of gestures in the West: third to thirteenth centuries. Bremmer, J. H. Roodenburg (eds) A Cultural history of gesture, pp. 59-70. Polity Press, Cambridge.

Scheuer, L. y S. Black. 2000. Developmental Juvenile Osteology. Academic Press, London.

Sillar, B. 1992. The social life of the Andean dead. Archaeological Review from Cambridge, 11(1): 107-123.

Sillar, B. 1996. The dead and the drying: techniques for transforming people and things in the Andes. Journal of Material Culture, 1: 259-289.

Synnott, A. 1993. The body social. Symbolism, self and society, Routledge, London.

Thomas, J. 2007 Archaeology's humanism and the materiality of the body. Insoll, T. (ed) The archaeology of identities, 13, pp. 211-224, Routledge, London.

Turner, T. 1995. Social body and embodied subject: bodiliness, subjectivity, and sociality among the Kayapo. Cultural Anthropology, 10(2): 143-170.

Ubelaker, D.H. 1989. Human skeletal remains. Taraxacum Press, Washington, D.C. 